\title{
Estimativas de parâmetros genéticos para produção e composição do leite de vacas da raça Holandesa no estado do Paraná
}

\section{Meiby Carneiro de Paula ${ }^{1}$, Elias Nunes Martins ${ }^{2}$, Luiz Otávio Campos da Silva ${ }^{3}$, Carlos Antonio Lopes de Oliveira ${ }^{4}$, Altair Antônio Valotto ${ }^{5}$, Eliane Gasparino ${ }^{2}$}

\author{
1 Pós-Graduação em Zootecnia da Universidade Estadual de Maringá - PR, Bolsista da CAPES. \\ 2 Departamento de Zootecnia da Universidade Estadual de Maringá - PR. \\ 3 EMBRAPA - Campo Grande. \\ ${ }^{4}$ Curso de Zootecnia da Universidade Estadual do Mato Grosso do Sul. \\ ${ }^{5}$ Serviço de Registro Genealógico da Associação Paranaense de Criadores de Bovinos da Raça Holandesa.
}

RESUMO - Estimaram-se as herdabilidades e as correlações genéticas para as produções de leite (PL), de gordura (PG) e de proteína (PP) e para os percentuais de gordura (\%G) e de proteína (\%P) no leite de vacas da raça Holandesa no estado do Paraná. Foram analisados 117.082 registros de lactações encerradas para estimativa das características PL, PG e PP e 116.747 registros de lactações para \%G e de \% P. Todos os animais foram controlados oficialmente pelo Serviço de Controle Leiteiro Mensal da Associação Paranaense de Criadores de Bovinos da Raça Holandesa entre janeiro de 1992 e dezembro de 2003. A estimação dos componentes de co-variância e dos parâmetros genéticos foi realizada por meio de inferência Bayesiana em análises uni e tricaracteres utilizando-se um modelo animal que incluiu os efeitos fixos de grupo de contemporâneos e número de ordenhas, além dos efeitos linear e quadrático da idade da vaca ao parto e os efeitos aleatórios genéticos aditivos, permanente de ambiente e residual. As médias e os respectivos desvios-padrão para as PL, PG e PP, em kg, foram 8.181,23 $\pm 1.903,51$; $270,88 \pm 67,58$ e $249,01 \pm 55,65$, respectivamente, e para os $\% \mathrm{G}$ e $\% \mathrm{P}$ foram 3,33 $\pm 0,45$ e 3,06 $\pm 0,19$, respectivamente As estimativas de herdabilidade para PL, PG, PP, \%G e \%P obtidas nas análises unicaracteres foram 0,$26 ; 0,28 ; 0,25 ; 0,60$ e 0,58 , respectivamente, e indicam que as características possuem variação genética aditiva que garante boa resposta à seleção. As estimativas das correlações genéticas entre PL e PG, PL e PP, PG e PP, PL e \%G, PL e \%P, \%G e \%P foram 0,56; 0,89; 0,$67 ;-0,42 ;-0,39$ e 0,56 , respectivamente, portanto, a seleção realizada para aumentar a PL promoverá aumento na PG e $\mathrm{PP}$ e redução nos \% $\mathrm{G}$ e $\mathrm{P}$.

Palavras-chave: amostrador de Gibbs, componentes do leite, correlação genética, herdabilidade, inferência Bayesiana

\section{Estimates of genetic parameters for yield and composition of milk of Holstein cows in Paraná State}

\begin{abstract}
The objectives of this study were to estimate heritabilities and genetic correlations for milk (MY), fat $(\mathrm{FY})$ and protein (PY) yields and for percentages of fat $(\% \mathrm{~F})$ and protein $(\% \mathrm{P})$ in milk of Holstein cows in Paraná State. Data consisted of 117,082 lactation records for MY, FY and PY and 116,747 records for \%F and \%P from herds supervised by the Milk Recording Service of the Holstein Breeders Association of Paraná from January 1992 to December 2003. Covariance components and genetic parameters were estimated by Bayesian inference using an animal model including the fixed effects of contemporary group, number of milking, linear and quadratic effects for calving age and the additive genetic, permanent environmental and residual random effects. Averages and respective standard deviations for MY, FY and PY (kg) were respectively $8,181.23 \pm 1,903.51,270.88 \pm 67.58$ and $249.01 \pm 55.65$ and for $\% \mathrm{~F}$ and $\% \mathrm{P}$ they were $3.33 \pm 0.45$ and 3.06 0.19 , respectively. Heritability estimates for MY, FY, PY, \% G and \% P obtained from single-trait analyses were $0.26,0.28$, $0.25,0.60$ and 0.58 , respectively. They indicate that these traits may be improved by selection. Genetic correlation estimates between MY and FY, MY and PY, FY and PY, MY and \%F, MY and \% P and for \%F and \%P were 0.56, 0.89, 0.67, - 0.42 , -0.39 and 0.56 , respectively. Selection for increasing MY will result in positive correlated genetic gains for $\mathrm{kg}$ of fat and protein but decrease their percentages in milk.
\end{abstract}

Key Words: Bayesian inference, genetic correlation, Gibbs sampler, heritability, milk components

Este artigo foi recebido em 18/1/2006 e aprovado em 11/10/2007.

Correspondências devem ser enviadas para meibydepaula@hotmail.com. 


\section{Introdução}

A pecuária leiteira no Paraná é reconhecida por seus rebanhos de alto potencial genético para produção e composição de leite. Para que possam ser aplicadas práticas efetivas de seleção para essas características, é necessário que parâmetros genéticos sejam estimados na própria população sob seleção. A correta estimação dos parâmetros genéticos é fundamental em programas de melhoramento genético, pois permite predizer o valor genético dos animais e identificar animais geneticamente superiores.

No Paraná, estimativas para as características de produção (Ribas et al., 1983; Ribas et al., 1993; Almeida et al., 1997; Falcão et al., 2006) e composição do leite (Almeida et al., 1997) têm sido obtidas, mas, em virtude das diferenças genéticas da população, de ambiente, do tipo de análise e do método de estimação de componentes de co-variância, essas estimativas têm variado consideravelmente.

Métodos Bayesianos têm sido utilizados para estimação dos componentes de co-variância e dos parâmetros genéticos para avaliação genética de animais (Gianola \& Fernando, 1986; Rekaya et al., 2003; Falcão et al., 2006). Esses métodos permitem a análise de grandes conjuntos de dados, não requerem soluções para as equações de modelos mistos e propiciam estimativas diretas e acuradas dos componentes de co-variância e dos parâmetros genéticos das características de interesse.

Os objetivos neste trabalho foram estimar as herdabilidades e as correlações genéticas para as produções de leite (PL), gordura (PG) e proteína (PP) e para os percentuais de gordura $(\% \mathrm{G})$ e proteína $(\% \mathrm{P})$ do leite em bovinos da raça Holandesa no estado do Paraná utilizando-se inferência Bayesiana.

\section{Material e Métodos}

Foram utilizados 195.886 registros de lactações encerradas de vacas da raça Holandesa, cujo controle foi realizado pelo Programa de Análise de Rebanhos Leiteiros do Paraná (PARLPR), desenvolvido em convênio com a As sociação Paranaense de Criadores de Bovinos da Raça Holandesa (APCBRH), localizada em Curitiba, Paraná. Os animais foram controlados oficialmente pelo Serviço de Controle Leiteiro Mensal da APCBRH entre janeiro de 1992 e dezembro de 2003.

Para garantir a consistência das informações, o conjunto de dados original foi editado no programa computacional SAS $^{\circledR}$ (Statistical Analysis System) (2000), impondo-se restrições que resultaram na eliminação de observações inconsistentes. Foram eliminadas informações de vacas cujos pais tinham menos de oito filhas e de rebanhos com menos de dez lactações. Definiram-se duas estações de parto, uma de abril a setembro e outra de outubro a março, e grupos de contemporâneos (GC) formados por lactações de mesmo rebanho, ano e estação de parição. Apenas informações de grupos de contemporâneos com dez observações ou mais foram mantidas.

Após a aplicação de todas as restrições, o conjunto de dados final permaneceu com 117.082 registros de primeira à décima lactação para as produções de leite (PL), gordura (PG) e proteína (PP), ajustados para os 305 dias de lactação de 49.676 vacas provenientes de 308 rebanhos, e com 116.747 registros de primeira à décima lactação para os percentuais de gordura $(\% \mathrm{G})$ e de proteína $(\% \mathrm{P})$ ajustados para os 305 dias de lactação, de 49.612 vacas provenientes de 308 rebanhos. Os rebanhos estavam distribuídos em sete bacias leiteiras do estado do Paraná: Castro, Carambeí, Witmarsum, Arapoti, Sul, Norte e Oeste.

A estimação dos componentes de co-variância e dos parâmetros genéticos foi realizada utilizando-se abordagem Bayesiana, por meio do programa MTGSAM (Multiple Trait Gibbs Sampling in Animal Models) desenvolvido por Van Tassel \& Van Vleck (1995). Primeiramente foram realizadas análises unicaracteres para características PL, PG, PP, \%G e \%P. Posteriormente, foram realizadas duas análises tricaracteres, uma envolvendo PL, PG e PP e outra envolvendo PL, \%G e \%P. Utilizou-se um modelo animal que incluiu os efeitos fixos de GC e número de ordenhas, os efeitos linear e quadrático da idade da vaca ao parto, em meses, e os efeitos aleatórios genético aditivo, permanente de ambiente e o erro residual.

Nas análises unicaracteres, utilizou-se o modelo animal a seguir:

$$
y=X \beta+Z u+e
$$

em que: $Z=\left[\begin{array}{ll}Z_{1} & Z_{2}\end{array}\right]$ e u $=\left[\begin{array}{l}a \\ p\end{array}\right]$ de modo que: $y$ é o vetor de observações; $X, Z_{1}$ e $Z_{2}$, as matrizes de incidência dos efeitos fixos, dos efeitos genéticos aditivos e dos efeitos permanentes de ambiente, respectivamente; e $\beta, a, p$ e $e$ são, respectivamente, os vetores dos efeitos fixos, genéticos aditivos, permanentes de ambiente e dos erros aleatórios associados a cada observação.

Para as análises tricaracteres, utilizou-se o modelo animal:

$y=\left[\begin{array}{l}y_{1} \\ y_{2} \\ y_{3}\end{array}\right]=\left[\begin{array}{ccc}X_{1} & 0 & 0 \\ 0 & X_{2} & 0 \\ 0 & 0 & X_{3}\end{array}\right]\left[\begin{array}{l}\beta_{1} \\ \beta_{2} \\ \beta_{3}\end{array}\right]+\left[\begin{array}{ccc}Z_{1_{1}} & 0 & 0 \\ 0 & Z_{1_{2}} & 0 \\ 0 & 0 & Z_{1_{3}}\end{array}\right] \cdot\left[\begin{array}{l}a_{1} \\ a_{2} \\ a_{3}\end{array}\right]+\left[\begin{array}{ccc}Z_{2_{1}} & 0 & 0 \\ 0 & Z_{2_{2}} & 0 \\ 0 & 0 & Z_{2_{3}}\end{array}\right] \cdot\left[\begin{array}{l}p_{1} \\ p_{2} \\ p_{3}\end{array}\right]+\left[\begin{array}{l}e_{1} \\ e_{2} \\ e_{3}\end{array}\right]$ 
em que: $y_{1}, y_{2}$ e $y_{3}$ são os vetores de observação para PL, PG e PP, ou para PL, \%G e \%P, respectivamente; $\beta_{1}, \beta_{2}$ e $\beta_{3}$, os vetores dos efeitos fixos; $a_{1}, a_{2}$ e $a_{3}$, os vetores dos efeitos genéticos aditivos; $p_{1}, p_{2}$ e $p_{3}$, os vetores dos efeitos permanentes de ambiente; $X_{1}, X_{2}$ e $X_{3}$, as matrizes de incidência dos efeitos fixos; $Z_{1_{1}}, Z_{1_{2}}$ e $Z_{1_{3}}$, as matrizes de incidência dos efeitos genéticos aditivos; $Z_{2_{1}}, Z_{2_{2}}$ e $Z_{2_{3}}$, as matrizes dos efeitos permanentes de ambiente; $e_{1}, e_{2} \mathrm{e}$ $e_{3}$, os valores dos erros aleatórios; e os índices 1, 2 e 3 são relativos à primeira, segunda e terceira características.

Assumiu-se uma distribuição inicial uniforme para os efeitos fixos. Nas análises unicaracteres, considerou-se que os componentes de variância genética aditiva $\left(\sigma_{a}^{2}\right)$, permanente de ambiente $\left(\sigma_{p}^{2}\right)$ e residual $\left(\sigma_{e}^{2}\right)$ apresentam distribuição Gama Invertida ( $\Gamma \mathrm{I})$, da forma: $\sigma_{a}^{2} \sim \Gamma \mathrm{I}\left(g_{0}, v_{g}\right)$, $\sigma_{p}^{2} \sim \Gamma \mathrm{I}\left(p_{0}, v_{p}\right)$ e $\sigma_{e}^{2} \sim \Gamma \mathrm{I}\left(s_{0}, v_{e}\right)$, em que:

$f\left(\sigma_{a}^{2} \mid g_{0}, v_{g}\right) \propto\left(\sigma_{a}^{2}\right)^{-\frac{1}{2}\left(v_{g}+2\right)} \cdot e^{-\frac{1}{2}\left(g_{g} \sigma_{a}^{-2}\right)}$

$f\left(\sigma_{p}^{2} \mid p_{0}, v_{p}\right) \propto\left(\sigma_{p}^{2}\right)^{-\frac{1}{2}\left(v_{p}+2\right)} \cdot e^{-\frac{1}{2}\left(p_{0} \sigma_{p}^{-2}\right)}$

$f\left(\sigma_{e}^{2} \mid s_{0}, v_{e}\right) \propto\left(\sigma_{e}^{2}\right)^{-\frac{1}{2}\left(v_{e}+2\right)} \cdot e^{-\frac{1}{2}\left(s_{0} \sigma_{e}^{-2}\right)}$

em que: $v_{\mathrm{g}}, v_{\mathrm{p}}$ e $v_{\mathrm{e}}$ são os graus de liberdade das distribuições, que equivalem ao grau de crença dos parâmetros $\sigma_{a}^{2}, \sigma_{p}^{2}$ e $\sigma_{e}^{2}$, respectivamente; $g_{0}, p_{0}$ e $s_{0}$ são os parâmetros escala das distribuições de $\sigma_{a}^{2}, \sigma_{p}^{2}$ e $\sigma_{e}^{2}$, respectivamente.

Nas análises tricaracteres, para os componentes de co-variância genética aditiva, permanente de ambiente e residual, considerou-se que $G, P$ e $R$ possuem distribuição de Wishart Invertida $(I W)$ na forma: $G \sim I W$ $\left(G_{o}, v_{g}\right), P \sim I W\left(P_{0}, v_{p}\right)$ e $R \sim I W\left(\mathrm{R}_{0}, v_{e}\right)$, em que:

$$
\begin{aligned}
& f\left(G \mid G_{o}, v_{g}\right) \propto|G|^{-\frac{1}{2}\left(v_{g}+m_{g}+1\right)} \cdot e^{-\frac{1}{2} \operatorname{tr}\left(G_{0}^{-1} G^{-1}\right)} \\
& f\left(P \mid P_{o}, v_{p}\right) \propto|P|^{-\frac{1}{2}\left(v_{p}+m_{p}+1\right)} \cdot e^{-\frac{1}{2} \operatorname{tr}\left(P_{0}^{-1} P^{-1}\right)} \\
& f\left(R \mid R_{o}, v_{e}\right) \propto|R|^{-\frac{1}{2}\left(v_{e}+m_{e}+1\right)} \cdot e^{-\frac{-1}{2} \operatorname{tr}\left(R_{0}^{-1} R^{-1}\right)}
\end{aligned}
$$

em que: $m_{g}, m_{p}$ e $m_{e}$ são as ordens das matrizes $G, P$ e $R$, respectivamente; e $G_{0}, P_{0}$ e $R_{O}$ são as matrizes de parâmetro escala das distribuições $G, P$ e $R$, respectivamente.

Tanto para as análises unicaracteres como para as tricaracteres, foram geradas cadeias de 120.000 iterações, com descarte inicial de 20.000 iterações e intervalo de amostragem de 100 iterações, resultando em 1.000 amostras dos componentes de co-variância para cada análise realizada. A monitoração da convergência das cadeias geradas pelo amostrador de Gibbs foi realizada por meio de análise gráfica e dos testes de diagnóstico de Geweke e de Heidelberger \& Welch, disponíveis no CODA (Convergence Diagnosis and Output Analysis), implementado no programa R (2004).

Foram construídos os intervalos de credibilidade para todos os componentes de co-variância e parâmetros genéticos estimados, a $90 \%$ de probabilidade.

\section{Resultados e Discussão}

As médias e os desvios-padrão para as produções de leite (PL), de gordura (PG) e de proteína (PP), em kg, ajustados para os 305 dias de lactação foram 8.181,23 $\pm 1.903,51$; $270,88 \pm 67,58$; e $249,01 \pm 55,65$, respectivamente, e para os percentuais de gordura $(\% \mathrm{G})$ e de proteína $(\% \mathrm{P})$ foram $3,33 \pm 0,45$ e $3,06 \pm 0,19$, respectivamente.

Houve indicação de convergência para todas as cadeias por meio de análise gráfica e da utilização dos testes de diagnóstico nas análises uni e tricaracteres.

As médias posteriores das estimativas de herdabilidade para PL, PG, PP, \% G e \% P obtidas nas análises unicaracteres foram iguais a 0,$26 ; 0,28 ; 0,25 ; 0,60$ e 0,58 , respectivamente, semelhantes às obtidas nas análises tricaracteres (Tabela 1).

As médias foram obtidas com grande precisão, pois apresentaram baixo erro-padrão e se mantiveram no intervalo de credibilidade calculado. Estes resultados indicam que as características estudadas possuem variação genética aditiva suficiente para boa resposta à seleção. Estimativas semelhantes foram obtidas por Chauhan \& Hayes (1991) utilizando o método da máxima verossimilhança restrita. Outros autores estimaram os parâmetros genéticos para características produtivas de bovinos da raça Holandesa utilizando REML e também encontraram valores semelhantes aos encontrados neste trabalho. As estimativas para PL, PG e \%G encontradas por Ribas et al. (1993) no estado do Paraná foram de $0,23 \pm 0,05 ; 0,28 \pm 0,05 ; \mathrm{e} 0,47 \pm 0,08$, respectivamente. Chauhan \& Hayes (1991) encontraram, para vacas em primeira lactação, valores de herdabilidade para PL, PG, PP, \% Ge \%P de 0,$29 ; 0,31 ; 0,25 ; 0,65$ e 0,61 , respectivamente. Os valores de herdabilidade estimados por Urioste et al. (2003) para PL foram de 0,$23 ; 0,24$ e 0,25 para a primeira, segunda e últimas lactações.

Os valores encontrados neste estudo para as características PL e PG foram superiores ao descritos por Ferreira \& Fernandes (2000), que estimaram os parâmetros genéticos para PL e PG utilizando o método da máxima verossimilhança 
Tabela 1 - Médias posteriores e erro-padrão dos componentes de variância ${ }^{1}$ e das herdabilidades $\left(h_{a}^{2}\right)$ para as produções de leite (PL), de gordura (PG) e de proteína (PP), em kg, e para os percentuais de gordura (\%G) e proteína (\%P) do leite, com seus respectivos intervalos de credibilidade (entre parênteses) no nível de $90 \%$, obtidos em análise tricaracter

\begin{tabular}{lccc}
\hline Estimativa $^{1}$ & $\mathrm{PL}$ & $\mathrm{PG}$ & $\mathrm{PP}$ \\
\hline$\sigma_{a}^{2}$ & $512.766 \pm 562(484.272-540.876)$ & $678,5 \pm 0,56(650,0-707,8)$ & $441,8 \pm 0,43(419,0-465,1)$ \\
$\sigma_{p}^{2}$ & $341.869 \pm 413(320.084-364.158)$ & $443,0 \pm 0,41(421,9-464,7)$ & $305,0 \pm 0,32(288,7-322,0)$ \\
$\sigma_{e}^{2}$ & $1.054 .146 \pm 180,98(1.044 .632-1.063 .276)$ & $1.313,5 \pm 0,22(1.302,1-1325,1)$ & $897,7 \pm 0,15(889,6-905,6)$ \\
$h_{a}^{2}$ & $0,27 \pm 0,0003(0,26-0,28)$ & $0,28 \pm 0,0002(0,27-0,29)$ & $0,27 \pm 0,0002(0,26-0,28)$ \\
\hline & $\mathrm{PL}$ & $\% \mathrm{G}$ & $\% \mathrm{P}$ \\
\hline$\sigma_{a}^{2}$ & $504.800 \pm 555(476.239-533.166)$ & $0,096 \pm 0,00006(0,092-0,099)$ & $0,017 \pm 0,000009(0,017-0,018)$ \\
$\sigma_{p}^{2}$ & $346.800 \pm 403(325.760-367.143)$ & $0,021 \pm 0,00003(0,020-0,023)$ & $0,003 \pm 0,00001(0,003-0,004)$ \\
$\sigma_{e}^{2}$ & $1.051 .754 \pm 178,06(1.042 .655-1.061 .322)$ & $0,042 \pm 0,000007(0,041-0,042)$ & $0,009 \pm 0,000002(0,008-0,009)$ \\
$h_{a}^{2}$ & $0,27 \pm 0,0003(0,25-0,28)$ & $0,60 \pm 0,0003(0,59-0,62)$ & $0,59 \pm 0,0002(0,58-0,60)$ \\
\hline
\end{tabular}

${ }_{1}^{1} \sigma_{a}^{2}, \sigma_{p}^{2}$ e $\sigma_{e}^{2}$ são as variâncias genética aditiva, de efeito permanente de ambiente e de efeito residual, respectivamente.

restrita sem derivadas (DFREML) e encontraram valores de herdabilidade iguais a 0,18 , tanto para PL como para a PG.

As correlações genéticas foram altas e positivas para PL e PG, PL e PP, PG e PP e para \%G e \%P e altas e negativas para PL e \%G e PL e \%P (Tabela 2), indicando a existência de associação genética entre essas características. A seleção para aumentar a PL promoverá aumento na PG e PP e redução nos \%G e \%P.

Correlações genéticas altas e positivas entre PL e PG também foram observadas por Chauhan \& Hayes (1991), 0,45, Ribas et al. (1993), 0,78, Almeida et al. (1997), 0,52, Ferreira \& Fernandes (2000), 0,98, Freitas et al. (2001), 0,86 a 1,00, e Weber et al. (2005), 0,91 a 1,00. Para PL e \%G, Chauhan \& Hayes (1991) e Ribas et al. (1993) também relataram correlações genéticas negativas, de -0,49 e -0,13, respectivamente.

Tabela 2 - Médias posteriores e erro-padrão das correlações genéticas entre a produção de leite $(\mathrm{PL})$ e as produções de gordura $(P G)$ e proteína (PP) do leite, em $\mathrm{kg}$, e entre a $\mathrm{PL}$ e os percentuais de gordura (\%G) e proteína $(\% \mathrm{P})$ do leite obtidas nas análises tricaracteres

\begin{tabular}{lccc}
\hline Característica & PL & PG & $\% \mathrm{G}$ \\
\hline PG & $0,56 \pm 0,0004$ & - & - \\
PP & $0,89 \pm 0,0001$ & $0,67 \pm 0,0003$ & - \\
$\% G$ & $-0,42 \pm 0,0005$ & - & - \\
$\% \mathrm{P}$ & $-0,39 \pm 0,0005$ & - & $0,56 \pm 0,0003$ \\
\hline
\end{tabular}

\section{Conclusões}

As produções de leite, gordura e proteína e os percentuais de gordura e proteína possuem variabilidade genética aditiva suficiente para justificar sua inclusão em programas de melhoramento de bovinos da raça Holandesa no estado do Paraná. A seleção para aumentar a produção de leite nesta população promoverá aumento nas produções de gordura e proteína e redução nos percentuais de gordura e proteína.

\section{Agradecimento}

À Associação Paranaense de Criadores de Bovinos da Raça Holandesa, pela concessão do banco de dados.

\section{Literatura Citada}

ALMEIDA, R.; RIBAS, N.P.; MONARDES, H.G. Estudo dos efeitos genéticos sobre as características produtivas de vacas da raça Holandesa na região da Batavo, Paraná. In: REUNIÃO ANUAL DA SOCIEDADE BRASILEIRA DE ZOOTECNIA, 34., 1997, Juiz de Fora. Anais... Juiz de Fora: Sociedade Brasileira de Zootecnia, 1997. v.3, p.68-70.

CHAUHAN, V.P.S.; HAYES, J.F. Genetic parameters for first lactation milk production and composition traits Holsteins using multivariate restricted maximum likelihood. Journal of Dairy Science, v.74, p.603-610, 1991.

FALCÃO, A.J.S.; MARTINS, E.N.; COSTA, C.N. et al. Heterocedasticidade entre estados para produção de leite em vacas da raça Holandesa usando métodos bayesianos via amostrador de Gibbs. Revista Brasileira de Zootecnia, v. 35 , n.2, p.405-414, 2006. 
FERREIRA, G.B.; FERNANDES, H.D. Parâmetros genéticos para características produtivas em bovinos da raça holandesa no Estado de Goiás. Revista Brasileira de Zootecnia, v.29, p.421-426, 2000 .

FREIT AS, A.F.; DURÃES, M.C.; VALENTE, J. et al. Parâmetros genéticos para produção de leite e gordura nas três primeiras lactações de vacas holandesas. Revista Brasileira de Zootecnia, v.30, n.3, p.709-713, 2001.

GIANOLA, D.; FERNANDO, R.L. Bayesian methods in animal breeding theory. Journal of Animal Science, v.63, p. 217-277, 1986.

R Development Core Team [2004]. R: a language and environment for statistical computing. $\mathrm{R}$ Foundation for Statistical Computing, Vienna, Austria. Disponível em: <http:/ /www.R-project.org.>

REKAYA, R.; WEIGEL, K.A.; GIANOLA, D. Bayesian estimation of parameters of a structural model for genetic covariances between milk yield in five regions of the United States. Journal of Dairy Science, v.86, p.1837-1844, 2003.

RIBAS, N.P.; MILAGRES, J.C.; GARCIA, J.A. et al. Estudo da produção de leite e gordura em rebanhos Holandeses da bacia leiteira de Castrolanda, Estado do Paraná. Revista Brasileira de Zootecnia, v.12, n.4, p.720-740, 1983.

RIBAS, N.P.; RORATO, P.R.N; LÔBO, R.B. et al. Estimativas de parâmetros genéticos para as características de produção da raça holandesa no Estado do Paraná. Revista Brasileira de Zootecnia, v.22, n.4, p.634-641, 1993.

STATISTICAL ANALYSIS SYSTEM - SAS. SAS/STAT ${ }^{\circledR}$. User's guide: statistics, versão 8.1. 4.ed., Cary: SAS Institute, 2000. V.2. (CD-ROM)

URIOSTE, J.I.; REKAYA, R.; GIANOLA, D. et al. Model comparison for genetic evaluation of milk yield in Uruguayan Holteins. Livestock Production Science, v.84, p.63-73, 2003.

Van TASSEL, C.P.; Van VLECK D.L.A manual for use of MTGSAM. A set of FORTRAN programs to apply Gibbs sampling to animal models for variance component estimation (DRAFT) . Lincoln: Department of Agriculture Research Service, 1995. 86p.

WEBER, T.; RORATO, P.R.N.; FERREIRA, G.B.B. et al. Coeficientes de herdabilidade e correlações genéticas para as produções de leite e de gordura, em diferentes níveis de produção, para a raça Holandesa no Estado do Rio Grande do Sul. Revista Brasileira de Zootecnia, v.34, n.2, p.514-519, 2005. 